\title{
$q$-Bernstein-Schurer-Kantorovich Operators
}

\author{
Mehmet Ali Özarslan and Tuba Vedi* \\ Dedicated to Professor Hari M Srivastava
}

${ }^{*}$ Correspondence:

tuba.vedi@emu.edu.tr

Eastern Mediterranean University,

Mersin 10, Gazimagusa, TRNC, Turkey

\begin{abstract}
In the present paper, we introduce the $q$-Bernstein-Schurer-Kantorovich operators. We give the Korovkin-type approximation theorem and obtain the rate of convergence of this approximation by means of the first and the second modulus of continuity. Moreover, we compute the order of convergence of the operators in terms of the elements of Lipschitz class functions and the modulus of continuity of the derivative of the function.

MSC: Primary 41A10; 41A25; secondary 41A36
\end{abstract}

Keywords: $q$-analysis; $q$-integral operator; positive linear operators; q-Bernstein operators; modulus of continuity

\section{Introduction}

Some authors have defined general sequences of linear positive operators where the classical sequences can be achieved as particular cases. For instance, Schurer [1] proposed the following generalization of Bernstein operators in 1962. Let $C[a, b]$ denote the space of a continuous function on $[a, b]$. For all $n \in \mathbb{N}, f \in C[0, p+1]$ and fixed $p \in \mathbb{N}_{0}=\{0,1,2, \ldots\}$, the Bernstein-Schurer operators are defined by (see also, [2])

$$
B_{n}^{p}(f ; x)=\sum_{r=0}^{n+p} f\left(\begin{array}{c}
r \\
n
\end{array}\right)\left(\begin{array}{c}
n+p \\
r
\end{array}\right) x^{r}(1-x)^{n+p-r}, \quad x \in[0,1] .
$$

In 1987, $q$-based Bernstein operators were defined and studied by Lupaş [3]. In 1996, another $q$-based Bernstein operator was proposed by Phillips [4]. Then the $q$-based operators have become an active research area (see [5-9] and [10]).

Muraru [11] introduced and investigated the $q$-Bernstein-Schurer operators. She obtained the Korovkin-type approximation theorem and the rate of convergence of the operators in terms of the first modulus of continuity. These operators were defined, for fixed $p \in \mathbb{N}_{0}$ and for all $x \in[0,1]$, by

$$
B_{n}^{p}(f ; q ; x)=\sum_{r=0}^{n+p} f\left(\frac{[r]}{[n]}\right)\left[\begin{array}{c}
n+p \\
r
\end{array}\right] x^{r} \prod_{s=0}^{n+p-r-1}\left(1-q^{s} x\right),
$$

where $0<q<1$. If we choose $p=0$ in (1.1), we get the classical $q$-Bernstein operators [4].

\section{Springer}

(c) 2013 Özarslan and Vedi; licensee Springer. This is an Open Access article distributed under the terms of the Creative Commons Attribution License (http://creativecommons.org/licenses/by/2.0), which permits unrestricted use, distribution, and reproduction in any medium, provided the original work is properly cited. 
Recall that for each nonnegative integer $r,[r]$ is defined as

$$
[r]= \begin{cases}\left(1-q^{r}\right) /(1-q), & q \neq 1 \\ r, & q=1\end{cases}
$$

and the $q$-factorial of the integer $r$ is defined by

$$
[r] != \begin{cases}{[r][r-1] \cdots[1],} & r=1,2,3, \ldots, \\ 1, & r=0 .\end{cases}
$$

For integers $n$ and $r$, with $0 \leq r \leq n, q$-binomial coefficients are defined by [12]

$$
\left[\begin{array}{l}
n \\
r
\end{array}\right]=\frac{[n] !}{[n-r] ![r] !} .
$$

Afterwards, several properties and results of the operators defined by (1.1), such as the order of convergence of these operators by means of Lipschitz class functions, the first and the second modulus of continuity and the rate of convergence of the approximation process in terms of the first modulus of continuity of the derivative of the function, were given by the authors [13]. On the other hand, $q$-Szasz-Schurer operators were discussed in [14].

Kantorovich considered the linear positive operators $K_{n}(f ; x): L_{1}[0,1] \rightarrow L_{1}[0,1]$ which are defined for $f \in L_{1}[0,1]$ as follows:

$$
K_{n}(f ; x)=(n+1) \sum_{k=0}^{n} p_{n, k}(x) \int_{k / n+1}^{(k+1) /(n+1)} f(u) d u
$$

where $p_{n, k}(x)=\left(\begin{array}{l}n \\ k\end{array}\right) x^{k}(1-x)^{n-k}$. After this definition, the integral variants of classical and general operators have attracted a great interest (see [15-18] and [19]).

In 2007, Dalmanoğlu defined Kantorovich-type $q$-Bernstein operators by [20]

$$
B_{n}^{*}(f ; q ; x)=[n+1] \sum_{k=0}^{n} q^{-k}\left[\begin{array}{l}
n \\
k
\end{array}\right] x^{k} \prod_{s=0}^{n-k-1}\left(1-q^{s} x\right) \int_{[k] /[n+1]}^{[k+1] /[n+1]} f(t) d_{q} t .
$$

Notice that, the $q$-Jackson integral is defined on the interval $[0, b]$ as follows:

$$
\int_{0}^{b} f(t) d_{q} t=(1-q) b \sum_{j=0}^{\infty} f\left(q^{j} b\right) q^{j}, \quad 0<q<1
$$

Then she obtained the first three moments and gave the rate of convergence of the approximation process in terms of the first modulus of continuity [20].

In our definition, the integral that we consider in the $q$-Schurer-Bernstein-Kantorovich operator is

$$
\int_{0}^{1}\left(\frac{[r]}{[n+1]}+\frac{1+(q-1)[r]}{[n+1]} t\right)^{k} d_{q} t, \quad k \in \mathbb{N}_{0}, 0<q<1 .
$$


So, throughout this paper, we will use the following results, which are computed directly by the tools of $q$-calculus.

Using (1.2), we can find the following results:

$$
\int_{0}^{1} d_{q} t=(1-q) \sum_{j=0}^{\infty} q^{j}=(1-q) \frac{1}{1-q}=1
$$

where $0<q<1$. On the other hand, by (1.2) and (1.3) we get

$$
\begin{aligned}
& \int_{0}^{1}\left(\frac{[r]}{[n+1]}+\frac{1+(q-1)[r]}{[n+1]} t\right) d_{q} t \\
& \quad=\frac{1+(q-1)[r]}{[n+1]} \int_{0}^{1} t d_{q} t+\frac{[r]}{[n+1]} \int_{0}^{1} d_{q} t \\
& \quad=\frac{1}{[n+1]}(1-q) \sum_{j=0}^{\infty} q^{2 j}+\frac{q[r]}{[n+1]}=\frac{1}{[n+1]}(1-q) \frac{1}{1-q^{2}}+\frac{q[r]}{[n+1]} \\
& \quad=\frac{1}{[2][n+1]}+2 \frac{q[r]}{[2][n+1]} .
\end{aligned}
$$

Since

$$
\int_{0}^{1} t^{2} d_{q} t=(1-q) \sum_{j=0}^{\infty} q^{2 j} q^{j}=(1-q) \frac{1}{1-q^{3}}=\frac{1}{1+q+q^{2}}=\frac{1}{[3]}
$$

we have

$$
\begin{aligned}
\int_{0}^{1} & \left(\frac{[r]}{[n+1]}+\frac{1+(q-1)[r]}{[n+1]} t\right)^{2} d_{q} t \\
& =\int_{0}^{1}\left(\frac{[r]^{2}}{[n+1]^{2}}+2 \frac{[r](1+(q-1)[r])}{[n+1]^{2}} t+\frac{(1+(q-1)[r])^{2}}{[n+1]^{2}} t^{2}\right) d_{q} t \\
& =\frac{1}{[n+1]^{2}}\left((1+(q-1)[r])^{2} \int_{0}^{1} t^{2} d_{q} t+2[r](1+(q-1)[r]) \int_{0}^{1} t d_{q} t+[r]^{2} \int_{0}^{1} d_{q} t\right) \\
& =\frac{1}{[n+1]^{2}}\left\{\left(1+\frac{2(q-1)}{[2]}+\frac{(q-1)^{2}}{[3]}\right)[r]^{2}+\left(\frac{2}{[2]}+\frac{2(q-1)}{[3]}\right)[r]+\frac{1}{[3]}\right\} .
\end{aligned}
$$

Recall that the first three moments of the $q$-Bernstein-Schurer operators were given by Muraru in [11] as follows.

Lemma 1.1 For the first three moments of $B_{n}^{p}(f ; q ; x)$ we have:

(i) $B_{n}^{p}(1 ; q ; x)=1$,

(ii) $B_{n}^{p}(t ; q ; x)=\frac{[n+p]}{[n]} x$,

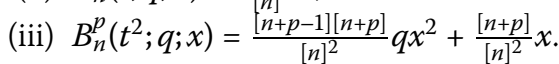

We organize the paper as follows.

Firstly, in section two, we define the $q$-Bernstein-Schurer-Kantorovich operators and obtain the moments of them. In section three, we obtain the rate of convergence of the $q$ Bernstein-Schurer-Kantorovich operators in terms of the first modulus of continuity. Also 
we give the order of approximation by means of Lipschitz class functions and the first and the second modulus of continuity. Furthermore, we compute the degree of convergence of the approximation process in terms of the first modulus of continuity of the derivative of the function.

\section{Construction of the operators}

For fixed $p \in \mathbb{N}_{0}$, we introduce the $q$-Bernstein-Schurer-Kantorovich operators $K_{n}^{p}(f ; q ; x)$ : $C[0, p+1] \rightarrow C[0,1]$

$$
K_{n}^{p}(f ; q ; x)=\sum_{r=0}^{n+p}\left[\begin{array}{c}
n+p \\
r
\end{array}\right] x^{r} \prod_{s=0}^{n+p-r-1}\left(1-q^{s} x\right) \int_{0}^{1} f\left(\frac{[r]}{[n+1]}+\frac{1+(q-1)[r]}{[n+1]} t\right) d_{q} t
$$

for any real number $0<q<1$, and $f \in C[0, p+1]$. It is clear that $K_{n}^{p}(f ; q ; x)$ is a linear and positive operator for $x \in[0,1]$.

For the first three moments and the first and the second central moment, we state the following lemma.

Lemma 2.1 For the q-Bernstein-Schurer-Kantorovich operators we have

(i)

$$
K_{n}^{p}(1 ; q ; x)=1,
$$

(ii)

$$
K_{n}^{p}(u ; q ; x)=\frac{2[n+p] q x+1}{[2][n+1]},
$$

(iii)

$$
\begin{aligned}
K_{n}^{p}\left(u^{2} ; q ; x\right)= & \frac{1}{[n+1]^{2}}\left\{\left(\frac{4 q^{4}+q^{3}+q^{2}}{[2][3]}\right)[n+p-1][n+p] x^{2}\right. \\
& \left.+\left(\frac{4 q^{3}+5 q^{2}+3 q}{[2][3]}\right)[n+p] x+\frac{1}{[3]}\right\},
\end{aligned}
$$

(iv)

$$
K_{n}^{p}((u-x) ; q ; x)=\left(2 \frac{[n+p]}{[2][n+1]} q-1\right) x+\frac{1}{[2][n+1]},
$$

(v)

$$
\begin{aligned}
K_{n}^{p}\left((u-x)^{2} ; q ; x\right)= & \left(\frac{4 q^{4}+q^{3}+q^{2}}{[2][3][n+1]^{2}}[n+p-1][n+p]-4 \frac{[n+p]}{[2][n+1]} q+1\right) x^{2} \\
& +\left(\frac{4 q^{3}+5 q^{2}+3 q}{[2][3][n+1]^{2}}[n+p]-\frac{2}{[2][n+1]}\right) x+\frac{1}{[3][n+1]^{2}} .
\end{aligned}
$$

Proof (i) From (1.3), we get

$$
K_{n}^{p}(1 ; q ; x)=\sum_{r=0}^{n+p}\left[\begin{array}{c}
n+p \\
r
\end{array}\right] x^{r} \prod_{s=0}^{n+p-r-1}\left(1-q^{s} x\right)=1
$$


(ii) Using (1.1), (1.4) and Lemma 1.1, we have

$$
\begin{aligned}
K_{n}^{p}(u ; q ; x)= & \sum_{r=0}^{n+p}\left[\begin{array}{c}
n+p \\
r
\end{array}\right] x^{r} \prod_{s=0}^{n+p-r-1}\left(1-q^{s} x\right)\left(\frac{1}{[2][n+1]}+\frac{2 q[r]}{[2][n+1]}\right) \\
= & \frac{1}{[2][n+1]} \sum_{r=0}^{n+p}\left[\begin{array}{c}
n+p] x^{r} \prod_{s=0}^{n+p-r-1}\left(1-q^{s} x\right) \\
r
\end{array}\right] \\
& +\sum_{r=0}^{n+p}[n+p] x^{r} \prod_{s=0}^{n+p-r-1}\left(1-q^{s} x\right) \frac{2 q[r]}{[2][n+1]} \frac{[n]}{[n]} \\
= & \frac{1}{[2][n+1]}+\frac{2 q[n]}{[2][n+1]} B_{n}^{q}(t ; q ; x) \\
= & \frac{1}{[2][n+1]}+\frac{2 q[n]}{[2][n+1]} \frac{[n+p]}{[n]} x \\
= & \frac{2[n+p] q x+1}{[2][n+1]}
\end{aligned}
$$

(iii) From (1.1), (1.4), (1.5) and then Lemma 1.1, we can calculate the $K_{n}^{p}\left(u^{2} ; q ; x\right)$ as follows:

$$
\begin{aligned}
& K_{n}^{p}\left(u^{2} ; q ; x\right) \\
& =\frac{1}{[n+1]^{2}}\left\{\sum_{r=0}^{n+p}\left[\begin{array}{c}
n+p \\
r
\end{array}\right] x^{r} \prod_{s=0}^{n+p-r-1}\left(1-q^{s} x\right)\left(1+\frac{2(q-1)}{[2]}+\frac{(q-1)^{2}}{[3]}\right)[r]^{2}\right. \\
& +\sum_{r=0}^{n+p}\left[\begin{array}{c}
n+p \\
r
\end{array}\right] x^{r} \prod_{s=0}^{n+p-r-1}\left(1-q^{s} x\right)\left(\frac{2}{[2]}+\frac{2(q-1)}{[3]}\right)[r] \\
& \left.+\sum_{r=0}^{n+p}\left[\begin{array}{c}
n+p \\
r
\end{array}\right] x^{r} \prod_{s=0}^{n+p-r-1}\left(1-q^{s} x\right) \frac{1}{[3]}\right\} \\
& =\frac{1}{[n+1]^{2}}\left\{\left(\frac{3 q-1}{[2]}+\frac{(q-1)^{2}}{[3]}\right) \sum_{r=0}^{n+p}\left[\begin{array}{c}
n+p \\
r
\end{array}\right] x^{r} \prod_{s=0}^{n+p-r-1}\left(1-q^{s} x\right)[r]^{2} \frac{[n]^{2}}{[n]^{2}}\right. \\
& +\left(\frac{2}{[2]}+\frac{2(q-1)}{[3]}\right) \sum_{r=0}^{n+p}\left[\begin{array}{c}
n+p \\
r
\end{array}\right] x^{r} \prod_{s=0}^{n+p-r-1}\left(1-q^{s} x\right) \frac{[r]}{[n]}[n] \\
& \left.+\frac{1}{[3]} \sum_{r=0}^{n+p}\left[\begin{array}{c}
n+p \\
r
\end{array}\right] x^{r} \prod_{s=0}^{n+p-r-1}\left(1-q^{s} x\right)\right\} \\
& =\frac{1}{[n+1]^{2}}\left\{\frac{1}{[3]}+\left(\frac{2}{[2]}+\frac{2(q-1)}{[3]}\right)[n] \sum_{r=0}^{n+p}\left[\begin{array}{c}
n+p \\
r
\end{array}\right] x^{r} \prod_{s=0}^{n+p-r-1}\left(1-q^{s} x\right) \frac{[r]}{[n]}\right. \\
& \left.+\left(\frac{3 q-1}{[2]}+\frac{(q-1)^{2}}{[3]}\right)[n]^{2} \sum_{r=0}^{n+p}\left[\begin{array}{c}
n+p \\
r
\end{array}\right] x^{r} \prod_{s=0}^{n+p-r-1}\left(1-q^{s} x\right) \frac{[r]^{2}}{[n]^{2}}\right\} \\
& =\frac{1}{[n+1]^{2}}\left\{\frac{1}{[3]}+\left(\frac{2}{[2]}+\frac{2(q-1)}{[3]}\right)[n] B_{n}^{p}(t ; q ; x)\right. \\
& \left.+\left(\frac{3 q-1}{[2]}+\frac{(q-1)^{2}}{[3]}\right)[n]^{2} B_{n}^{p}\left(t^{2} ; q ; x\right)\right\} \text {. }
\end{aligned}
$$


Finally, we get

$$
\begin{aligned}
K_{n}^{p}\left(u^{2} ; q ; x\right)= & \frac{1}{[n+1]^{2}}\left\{\left(\frac{4 q^{4}+q^{3}+q^{2}}{[2][3]}\right)[n+p-1][n+p] x^{2}\right. \\
& \left.+\left(\frac{4 q^{3}+5 q^{2}+3 q}{[2][3]}\right)[n+p] x+\frac{1}{[3]}\right\},
\end{aligned}
$$

where $B_{n}^{p}(t ; q ; x)$ and $B_{n}^{p}\left(t^{2} ; q ; x\right)$ are the corresponding moments of the $q$-BernsteinSchurer operators.

(iv) It is obvious that

$$
K_{n}^{p}((u-x) ; q ; x)=K_{n}^{p}(u ; q ; x)-x K_{n}^{p}(1 ; q ; x)=\left(2 \frac{[n+p]}{[2][n+1]} q-1\right) x+\frac{1}{[2][n+1]}
$$

(v) Direct calculations yield,

$$
\begin{aligned}
& K_{n}^{p}\left((u-x)^{2} ; q ; x\right) \\
&=K_{n}^{p}\left(u^{2} ; q ; x\right)-2 x K_{n}^{p}(u ; q ; x)+x^{2} K_{n}^{p}(1 ; q ; x) \\
&=\frac{1}{[n+1]^{2}}\left\{\left(\frac{4 q^{4}+q^{3}+q^{2}}{[2][3]}\right)[n+p-1][n+p] x^{2}\right. \\
&\left.+\left(\frac{4 q^{3}+5 q^{2}+3 q}{[2][3]}\right)[n+p] x+\frac{1}{[3]}\right\}-2 x \frac{2[n+p] q x+1}{[n+1][2]}+x^{2} \\
&=\left(\frac{4 q^{4}+q^{3}+q^{2}}{[2][3][n+1]^{2}}[n+p-1][n+p]-4 \frac{[n+p]}{[2][n+1]} q+1\right) x^{2} \\
&+\left(\frac{4 q^{3}+5 q^{2}+3 q}{[2][3][n+1]^{2}}[n+p]-\frac{2}{[2][n+1]}\right) x+\frac{1}{[3][n+1]^{2}} .
\end{aligned}
$$

By Korovkin's theorem, we can state the following theorem.

Theorem 2.2 For all $f \in C[0, p+1]$, we have

$$
\lim _{n \rightarrow \infty}\left\|K_{n}^{p}\left(f ; q_{n}, \cdot\right)-f(\cdot)\right\|_{C[0,1]}=0
$$

provided that $q:=q_{n}$ with $\lim _{n \rightarrow \infty} q_{n}=1$ and that $\lim _{n \rightarrow \infty} \frac{1}{[n]}=0$.

\section{Rate of convergence}

In this section, we compute the rate of convergence of the operators in terms of the modulus of continuity, elements of Lipschitz classes and the first and the second modulus of continuity of the function. Furthermore, we calculate the rate of convergence in terms of the first modulus of continuity of the derivative of the function.

Now, we give the rate of convergence of the operators by means of the first modulus of continuity. Recall that the first modulus of continuity of $f$ on the interval $C[0, p+1]$ for $\delta>0$ is given by

$$
\omega(f, \delta)=\max _{\substack{|h|<\delta \\ x, x+h \in[0, p+1]}}\left|\Delta_{h} f(x)\right|=\max _{\substack{|h|<\delta \\ x, x+h \in[0, p+1]}}|f(x+h)-f(x)|
$$


or equivalently,

$$
\omega(f, \delta)=\max _{\substack{|t-x|<\delta \\ t, x \in[0, p+1]}}|f(t)-f(x)| .
$$

It is known that for all $f \in C[0, p+1]$, we have

$$
\lim _{\delta \rightarrow 0^{+}} \omega(f, \delta)=0
$$

and for any $\delta>0$,

$$
|f(x)-f(y)| \leq \omega(f, \delta)\left(\frac{|x-y|}{\delta}+1\right) .
$$

Theorem 3.1 Let $0<q<1$. Iff $\in C[0, p+1]$, we have

$$
\left|K_{n}^{p}(f ; q ; x)-f(x)\right| \leq 2 \omega\left(f, \sqrt{\delta_{n, q}(x)}\right)
$$

where $\omega(f, \cdot)$ is the modulus of continuity off and $\delta_{n, q}(x):=K_{n}^{p}\left((u-x)^{2} ; q ; x\right)$, which is given as Lemma 2.1.

Proof Using the linearity and positivity of the operator, we get

$$
\begin{aligned}
& \left|K_{n}^{p}(f ; q ; x)-f(x)\right| \\
& =\left|\sum_{r=0}^{n+p}\left[\begin{array}{c}
n+p \\
r
\end{array}\right] x^{r} \prod_{s=0}^{n+p-r-1}\left(1-q^{s} x\right) \int_{0}^{1}\left(f\left(\frac{[r]}{[n+1]}+\frac{1+(q-1)[r]}{[n+1]} t\right)-f(x)\right) d_{q} t\right| \\
& \leq \sum_{r=0}^{n+p}\left[\begin{array}{c}
n+p \\
r
\end{array}\right] x^{r} \prod_{s=0}^{n+p-r-1}\left(1-q^{s} x\right) \int_{0}^{1}\left|f\left(\frac{[r]}{[n+1]}+\frac{1+(q-1)[r]}{[n+1]} t\right)-f(x)\right| d_{q} t \\
& \leq \sum_{r=0}^{n+p} \int_{0}^{1}\left(\frac{\left|\frac{[r]}{[n+1]}+\frac{1+(q-1)[r]}{[n+1]} t-x\right|}{\delta}+1\right) \omega(f, \delta)\left[\begin{array}{c}
n+p \\
r
\end{array}\right] x^{r} \prod_{s=0}^{n+p-r-1}\left(1-q^{s} x\right) d_{q} t \\
& =\omega(f, \delta)\left(\sum_{r=0}^{n+p}\left[\begin{array}{c}
n+p \\
r
\end{array}\right] x^{r} \prod_{s=0}^{n+p-r-1}\left(1-q^{s} x\right)\right) \\
& +\frac{\omega(f, \delta)}{\delta}\left(\sum_{r=0}^{n+p} \int_{0}^{1}\left|\frac{[r]}{[n+1]}+\frac{1+(q-1)[r]}{[n+1]} t-x\right|\left[\begin{array}{c}
n+p \\
r
\end{array}\right] x^{r} \prod_{s=0}^{n+p-r-1}\left(1-q^{s} x\right) d_{q} t\right) .
\end{aligned}
$$

By the Cauchy-Schwarz inequality,

$$
\begin{aligned}
& \int_{0}^{1}\left|\left(\frac{[r]}{[n+1]}+\frac{1+(q-1)[r]}{[n+1]} t\right)-x\right| d_{q} t \\
& \quad \leq\left\{\int_{0}^{1}\left(\frac{[r]}{[n+1]}+\frac{1+(q-1)[r]}{[n+1]} t-x\right)^{2} d_{q} t\right\}^{\frac{1}{2}}\left\{\int_{0}^{1} 1 d_{q} t\right\}^{\frac{1}{2}} \\
& \quad=\left\{\int_{0}^{1}\left(\frac{[r]}{[n+1]}+\frac{1+(q-1)[r]}{[n+1]} t-x\right)^{2} d_{q} t\right\}^{\frac{1}{2}}:=\left\{a_{n, r}(x)\right\}^{\frac{1}{2}} .
\end{aligned}
$$


Now we have

$$
\left|K_{n}^{p}(f ; q ; x)-f(x)\right| \leq \omega(f, \delta)+\frac{\omega(f, \delta)}{\delta} \sum_{r=0}^{n+p}\left\{a_{n, r}(x)\right\}^{\frac{1}{2}} p_{n, r}(q ; x),
$$

where $p_{n, r}(q ; x)=\left[\begin{array}{c}n+p \\ r\end{array}\right] x^{r} \prod_{s=0}^{n+p-r-1}\left(1-q^{s} x\right)$. Again applying the Cauchy-Schwarz inequality, we get

$$
\begin{aligned}
& \left|K_{n}^{p}(f ; q ; x)-f(x)\right| \\
& \quad \leq \omega(f, \delta)+\frac{\omega(f, \delta)}{\delta}\left\{\sum_{r=0}^{n+p} a_{n, r}(x) p_{n, r}(q ; x)\right\}^{\frac{1}{2}}\left\{\sum_{r=0}^{n+p} p_{n, r}(q ; x)\right\}^{\frac{1}{2}} \\
& \quad=\omega(f, \delta)+\frac{\omega(f, \delta)}{\delta}\left\{\sum_{r=0}^{n+p} p_{n, r}(q ; x) \int_{0}^{1}\left(\frac{[r]}{[n+1]}+\frac{1+(q-1)[r]}{[n+1]} t-x\right)^{2} d_{q} t\right\}^{\frac{1}{2}} \\
& \quad=\omega(f, \delta)+\frac{\omega(f, \delta)}{\delta}\left[K_{n}^{p}\left((u-x)^{2} ; q ; x\right)\right]^{\frac{1}{2}} .
\end{aligned}
$$

So, we have

$$
\left|K_{n}^{p}(f ; q ; x)-f(x)\right| \leq \omega(f, \delta)+\frac{\omega(f, \delta)}{\delta}\left\{K_{n}^{p}\left((u-x)^{2} ; q ; x\right)\right\}^{1 / 2}
$$

Choosing $\delta: \delta_{n, q}(x)=K_{n}^{p}\left((u-x)^{2} ; q ; x\right)$, we obtain

$$
\left|K_{n}^{p}(f ; q ; x)-f(x)\right| \leq 2 \omega\left(f, \sqrt{K_{n}^{p}\left((u-x)^{2} ; q ; x\right)}\right) .
$$

The proof is concluded.

Now we give the rate of convergence of the operators $K_{n}^{p}$ in terms of the Lipschitz class $\operatorname{Lip}_{M}(\alpha)$, for $0<\alpha \leq 1$. Note that a function $f \in C[0, p+1]$ belongs to $\operatorname{Lip}_{M}(\alpha)$ if

$$
|f(t)-f(x)| \leq M|t-x|^{\alpha} \quad(t, x \in[0, p+1])
$$

is satisfied.

Theorem 3.2 Let $f \in \operatorname{Lip}_{M}(\alpha)$, then

$$
\left|K_{n}^{p}(f ; q ; x)-f(x)\right| \leq M\left(\delta_{n, q}(x)\right)^{\frac{\alpha}{2}}
$$

where $\delta_{n, q}(x)$ is the same as in Theorem 3.1.

Proof By the linearity and positivity, we have

$$
\begin{aligned}
& \left|K_{n}^{p}(f ; q ; x)-f(x)\right| \\
& \quad \leq \sum_{r=0}^{n+p}\left[\begin{array}{c}
n+p \\
r
\end{array}\right] x^{r} \prod_{s=0}^{n+p-r-1}\left(1-q^{s} x\right) \int_{0}^{1}\left|f\left(\frac{[r]}{[n+1]}+\frac{1+(q-1)[r]}{[n+1]} t\right)-f(x)\right| d_{q} t .
\end{aligned}
$$


Considering (3.2) and then applying the Hölder's inequality with $p=\frac{2}{\alpha}$ and $q=\frac{2}{2-\alpha}$, we get

$$
\begin{aligned}
& \int_{0}^{1}\left|f\left(\frac{[r]}{[n+1]}+\frac{1+(q-1)[r]}{[n+1]} t\right)-f(x)\right| d_{q} t \\
& \quad \leq M \int_{0}^{1}\left|\frac{[r]}{[n+1]}+\frac{1+(q-1)[r]}{[n+1]} t-x\right|^{\alpha} d_{q} t \\
& \quad \leq M\left\{\int_{0}^{1}\left(\frac{[r]}{[n+1]}+\frac{1+(q-1)[r]}{[n+1]} t-x\right)^{2} d_{q} t\right\}^{\frac{\alpha}{2}}\left\{\int_{0}^{1} 1 d_{q} t\right\}^{\frac{2-\alpha}{2}} \\
& \quad=M\left\{\int_{0}^{1}\left(\frac{[r]}{[n+1]}+\frac{1+(q-1)[r]}{[n+1]} t-x\right)^{2} d_{q} t\right\}^{\frac{\alpha}{2}}=M\left\{a_{n, r}(x)\right\}^{\frac{\alpha}{2}} .
\end{aligned}
$$

So, we have

$$
\left|K_{n}^{p}(f ; q ; x)-f(x)\right| \leq M \sum_{r=0}^{n+p}\left\{a_{n, r}(x)\right\}^{\frac{\alpha}{2}} p_{n, r}(q ; x),
$$

where $p_{n, r}(q ; x)=\left[\begin{array}{c}n+p \\ r\end{array}\right] x^{r} \prod_{s=0}^{n+p-r-1}\left(1-q^{s} x\right)$. Again applying Hölder's inequality with $p=\frac{2}{\alpha}$ and $q=\frac{2}{2-\alpha}$, we get

$$
\begin{aligned}
& \left|K_{n}^{p}(f ; q ; x)-f(x)\right| \\
& \quad \leq M\left\{\sum_{r=0}^{n+p} a_{n, r}(x) p_{n, r}(q ; x)\right\}^{\frac{\alpha}{2}}\left\{\sum_{r=0}^{n+p} 1 \cdot p_{n, r}(q ; x)\right\}^{\frac{2-\alpha}{2}} \\
& \quad=M\left\{\sum_{r=0}^{n+p} p_{n, r}(q ; x) \int_{0}^{1}\left(\frac{[r]}{[n+1]}+\frac{1+(q-1)[r]}{[n+1]} t-x\right)^{2} d_{q} t\right\}^{\frac{\alpha}{2}} \\
& \quad=M\left[K_{n}^{p}\left((u-x)^{2} ; q ; x\right)\right]^{\frac{\alpha}{2}} .
\end{aligned}
$$

Hence, the desired result is obtained.

Now let us denote by $C^{2}[0, p+1]$ the space of all functions $f \in C[0, p+1]$ such that $f^{\prime}, f^{\prime \prime} \in C[0, p+1]$. Let $\|f\|$ denote the usual supremum norm of $f$. The classical Peetre's $K$-functional and the second modulus of smoothness of the function $f \in C[0, p+1]$ are defined, respectively, by

$$
K(f, \delta):=\inf _{g \in C^{2}[0, p+1]}\left[\|f-g\|+\delta\left\|g^{\prime \prime}\right\|\right]
$$

and

$$
\omega_{2}(f, \delta):=\sup _{\substack{0<h<\delta, x, x+h \in[0, p+1]}}|f(x+2 h)-2 f(x+h)+f(x)|,
$$

where $\delta>0$. It is known that [21, p.177] there exists a constant $A>0$ such that

$$
K(f, \delta) \leq A \omega_{2}(f, \sqrt{\delta})
$$


Theorem 3.3 Let $q \in(0,1), x \in[0,1]$ and $f \in C[0, p+1]$. Then, for fixed $p \in \mathbb{N}_{0}$, we have

$$
\left|K_{n}^{p}(f ; q ; x)-f(x)\right| \leq C \omega_{2}\left(f, \sqrt{\alpha_{n, q}(x)}\right)+\omega\left(f, \beta_{n, q}(x)\right)
$$

for some positive constant $C$, where

$$
\begin{aligned}
\alpha_{n, q}(x):= & \left(4 \frac{[n+p]^{2}}{[2]^{2}[n+1]^{2}} q^{2}+\frac{4 q^{4}+q^{3}+q^{2}}{[2][3][n+1]^{2}}[n+p-1][n+p]-8 \frac{[n+p]}{[2][n+1]} q+2\right) x^{2} \\
& +\left(\frac{4 q^{3}+5 q^{2}+3 q}{[2][3][n+1]^{2}}[n+p]+4 \frac{[n+p]}{[2]^{2}[n+1]^{2}} q-\frac{4}{[2][n+1]}\right) x \\
& +\frac{1}{[3][n+1]^{2}}+\frac{1}{[2]^{2}[n+1]^{2}}
\end{aligned}
$$

and

$$
\beta_{n, q}(x):=\left(2 \frac{[n+p]}{[2][n+1]} q-1\right) x+\frac{1}{[2][n+1]} .
$$

Proof Define an auxiliary operator $K_{n, p}^{*}(f ; q ; x): C[0, p+1] \rightarrow C[0,1]$ by

$$
K_{n, p}^{*}(f ; q ; x):=K_{n}^{p}(f ; q ; x)-f\left(\frac{1}{[2][n+1]}(2[n+p] q x+1)\right)+f(x)
$$

Then, by Lemma 2.1, we get

$$
\begin{aligned}
& K_{n, p}^{*}(1 ; q ; x)=1, \\
& K_{n, p}^{*}((u-x) ; q ; x)=0 .
\end{aligned}
$$

Then, for a given $g \in C^{2}[0, p+1]$, it follows by the Taylor formula that

$$
g(y)-g(x)=(y-x) g^{\prime}(x)+\int_{x}^{y}(y-u) g^{\prime \prime}(u) d u, \quad y \in[0,1]
$$

Taking into account (3.7) and using (3.7), we get, for every $x \in(0,1)$, that

$$
\begin{aligned}
& \left|K_{n, p}^{*}(g ; q ; x)-g(x)\right| \\
& \quad=\left|K_{n, p}^{*}(g(y)-g(x) ; q ; x)\right| \\
& \quad=\left|g^{\prime}(x) K_{n, p}^{*}((u-x) ; q ; x)+K_{n, p}^{*}\left(\int_{x}^{y}(y-u) g^{\prime \prime}(u) d u ; q ; x\right)\right| \\
& \quad=\left|K_{n, p}^{*}\left(\int_{x}^{y}(y-u) g^{\prime \prime}(u) d u ; q ; x\right)\right| .
\end{aligned}
$$

Then by (3.6),

$$
\begin{aligned}
& \left|K_{n, p}^{*}(g ; q ; x)-g(x)\right| \\
& \quad=\left|K_{n, p}\left(\int_{x}^{y}(y-u) g^{\prime \prime}(u) d u ; q ; x\right)-\int_{x}^{\frac{2[n+p] q x+1}{[2][n+1]}}\left(\frac{2[n+p] q x+1}{[n+1][2]}-u\right) g^{\prime \prime}(u) d u\right|
\end{aligned}
$$




$$
\begin{aligned}
\leq & \left|K_{n, p}\left(\int_{x}^{y}(y-u) g^{\prime \prime}(u) d u ; q ; x\right)\right| \\
& +\left|\int_{x}^{\frac{2[n+p] q x+1}{[2][n+1]}}\left(\frac{2[n+p] q x+1}{[n+1][2]}-u\right) g^{\prime \prime}(u) d u\right| .
\end{aligned}
$$

Since

$$
\left|K_{n}^{p}\left(\int_{x}^{y}(y-u) g^{\prime \prime}(u) d u ; q ; x\right)\right| \leq\left\|g^{\prime \prime}\right\| K_{n}^{p}\left((y-x)^{2} ; q ; x\right)
$$

and

$$
\left|\int_{x}^{\frac{2[n+p] q x+1}{[2][n+1]}}\left(\frac{2[n+p] q x+1}{[2][n+1]}-u\right) g^{\prime \prime}(u) d u\right| \leq\left\|g^{\prime \prime}\right\|\left(\left(2 \frac{[n+p]}{[2][n+1]} q-1\right) x+\frac{1}{[2][n+1]}\right)^{2},
$$

we get

$$
\begin{aligned}
& \left|K_{n, p}^{*}(g ; q ; x)-g(x)\right| \\
& \quad \leq\left\|g^{\prime \prime}\right\| K_{n}^{p}\left((y-x)^{2} ; q ; x\right)+\left\|g^{\prime \prime}\right\|\left(\left(2 \frac{[n+p]}{[2][n+1]} q-1\right) x+\frac{1}{[2][n+1]}\right)^{2} .
\end{aligned}
$$

Hence Lemma 2.1 implies that

$$
\begin{aligned}
\left|K_{n, p}^{*}(g ; q ; x)-g(x)\right| \\
\leq\left\|g^{\prime \prime}\right\|\left[x^{2}\left(\frac{4 q^{4}+q^{3}+q^{2}}{[2][3][n+1]^{2}}[n+p-1][n+p]-4 \frac{[n+p]}{[2][n+1]} q+1\right)\right. \\
\quad+x\left(\frac{4 q^{3}+5 q^{2}+3 q}{[2][3][n+1]^{2}}[n+p]-\frac{2}{[2][n+1]}\right)+\frac{1}{[3][n+1]^{2}} \\
\left.\quad+\left(\left(2 \frac{[n+p]}{[2][n+1]} q-1\right) x+\frac{1}{[2][n+1]}\right)^{2}\right] .
\end{aligned}
$$

Since $\left\|K_{n, p}^{*}(f ; q ; \cdot)\right\| \leq 3$, considering (3.4) and (3.5), for all $f \in C[0, p+1]$ and $g \in C^{2}[0, p+$ $1]$, we may write from (3.8) that

$$
\begin{aligned}
\left|K_{n}^{p}(f ; q ; x)-f(x)\right| \leq & \left|K_{n, p}^{*}(f-g ; q ; x)-(f-g)(x)\right| \\
& +\left|K_{n, p}^{*}(g ; q ; x)-g(x)\right|+\left|f\left(\frac{2[n+p] q x+1}{[2][n+1]}\right)-f(x)\right| \\
\leq & 4\|f-g\|+\alpha_{n, q}(x)\left\|g^{\prime \prime}\right\|+\left|f\left(\frac{2[n+p] q x+1}{[2][n+1]}\right)-f(x)\right| \\
\leq & 4\left(\|f-g\|+\alpha_{n, q}(x)\left\|g^{\prime \prime}\right\|\right)+\omega\left(f, \beta_{n, q}(x)\right),
\end{aligned}
$$

which yields that

$$
\begin{aligned}
\left|K_{n}^{p}(f ; q ; x)-f(x)\right| & \leq 4 K\left(f, \alpha_{n, q}(x)\right)+\omega\left(f, \beta_{n, q}(x)\right) \\
& \leq C \omega_{2}\left(f, \sqrt{\alpha_{n, q}(x)}\right)+\omega\left(f, \beta_{n, q}(x)\right),
\end{aligned}
$$


where

$$
\begin{aligned}
\alpha_{n, q}(x):= & {\left[\left(4 \frac{[n+p]^{2}}{[2]^{2}[n+1]^{2}} q^{2}+\frac{4 q^{4}+q^{3}+q^{2}}{[2][3][n+1]^{2}}[n+p-1][n+p]-8 \frac{[n+p]}{[2][n+1]} q+2\right) x^{2}\right.} \\
& +x\left(\frac{4 q^{3}+5 q^{2}+3 q}{[2][3][n+1]^{2}}[n+p]+4 \frac{[n+p]}{[2]^{2}[n+1]^{2}} q-\frac{4}{[2][n+1]}\right) \\
& \left.+\frac{1}{[3][n+1]^{2}}+\frac{1}{[2]^{2}[n+1]^{2}}\right]
\end{aligned}
$$

and

$$
\beta_{n, q}(x):=\left(2 \frac{[n+p]}{[2][n+1]} q-1\right) x+\frac{1}{[2][n+1]} .
$$

Hence we get the result.

Now, we compute the rate of convergence of the operators $K_{n}^{p}$ in terms of the modulus of continuity of the derivative of the function.

Theorem 3.4 Let $0<q<1$ and $p \in \mathbb{N}_{0}$ be fixed. If $f(x)$ has a continuous derivative $f^{\prime}(x)$ and $\omega\left(f^{\prime}, \delta\right)$ is the modulus of continuity of $f^{\prime}(x)$ on $[0, p+1]$, then

$$
\left|K_{n}^{p}(f ; q ; x)-f(x)\right| \leq M \rho_{n, q}(x)+\omega\left(f^{\prime}, \delta\right)\left(1+\sqrt{\delta_{n, q, p}(x)}\right),
$$

where $M$ is a positive constant such that $\left|f^{\prime}(x)\right| \leq M(0 \leq x \leq p+1)$,

$$
\begin{aligned}
\delta_{n, q, p}(x) & \\
= & {\left[\left(\frac{4 q^{4}+q^{3}+q^{2}}{[2][3][n+1]^{2}}[n+p-1][n+p]-4 \frac{[n+p]}{[2][n+1]} q+1\right) x^{2}\right.} \\
& \left.+\left(\frac{4 q^{3}+5 q^{2}+3 q}{[2][3][n+1]^{2}}[n+p]-\frac{2}{[2][n+1]}\right) x+\frac{1}{[3][n+1]^{2}}\right]
\end{aligned}
$$

and

$$
\rho_{n, q}(x)=\left(2 \frac{[n+p]}{[2][n+1]} q-1\right) x+\frac{1}{[2][n+1]} .
$$

Proof Using the mean value theorem, we have

$$
\begin{aligned}
f( & \left.\frac{[r]}{[n+1]}+\frac{1+(q-1)[r]}{[n+1]} t\right)-f(x) \\
= & \left(\frac{[r]}{[n+1]}+\frac{1+(q-1)[r]}{[n+1]} t-x\right) f^{\prime}(\xi) \\
= & \left(\frac{[r]}{[n+1]}+\frac{1+(q-1)[r]}{[n+1]} t-x\right) f^{\prime}(x) \\
& +\left(\frac{[r]}{[n+1]}+\frac{1+(q-1)[r]}{[n+1]} t-x\right)\left(f^{\prime}(\xi)-f^{\prime}(x)\right),
\end{aligned}
$$


where $x<\xi<\frac{[r]}{[n+1]}+\frac{1+(q-1)[r]}{[n+1]} t$. Hence, we have

$$
\begin{aligned}
& \left|K_{n}^{p}(f ; q ; x)-f(x)\right| \\
& =\mid f^{\prime}(x) \sum_{r=0}^{n+p} \int_{0}^{1}\left(\frac{[r]}{[n+1]}+\frac{1+(q-1)[r]}{[n+1]} t-x\right)\left[\begin{array}{c}
n+p \\
r
\end{array}\right] x^{r} \prod_{s=0}^{n+p-r-1}\left(1-q^{s} x\right) d_{q} t \\
& +\sum_{r=0}^{n+p} \int_{0}^{1}\left(\frac{[r]}{[n+1]}+\frac{1+(q-1)[r]}{[n+1]} t-x\right) \\
& \times\left(f^{\prime}(\xi)-f^{\prime}(x)\right)\left[\begin{array}{c}
n+p \\
r
\end{array}\right] x^{r} \prod_{s=0}^{n+p-r-1}\left(1-q^{s} x\right) d_{q} t \mid \\
& \leq\left|f^{\prime}(x)\right| K_{n}^{p}((u-x) ; q ; x) \\
& +\sum_{r=0}^{n+p} \int_{0}^{1}\left(\frac{[r]}{[n+1]}+\frac{1+(q-1)[r]}{[n+1]} t-x\right) \\
& \times\left|f^{\prime}(\xi)-f^{\prime}(x)\right|\left[\begin{array}{c}
n+p \\
r
\end{array}\right] x^{r} \prod_{s=0}^{n+p-r-1}\left(1-q^{s} x\right) d_{q} t \\
& \leq M \rho_{n, q}(x) \\
& +\sum_{r=0}^{n+p} \int_{0}^{1}\left(\frac{[r]}{[n+1]}+\frac{1+(q-1)[r]}{[n+1]} t-x\right) \\
& \times\left|f^{\prime}(\xi)-f^{\prime}(x)\right|\left[\begin{array}{c}
n+p \\
r
\end{array}\right] x^{r} \prod_{s=0}^{n+p-r-1}\left(1-q^{s} x\right) d_{q} t
\end{aligned}
$$

where $\rho_{n, q}(x)$ is given in (3.9). Hence,

$$
\begin{aligned}
& \left|K_{n}^{p}(f ; q ; x)-f(x)\right| \\
& \leq M \rho_{n, q}(x)+\sum_{r=0}^{n+p} \int_{0}^{1} \omega\left(f^{\prime}, \delta\right)\left(\frac{\left(\frac{[r]}{[n+1]}+\frac{1+(q-1)[r]}{[n+1]} t-x\right)}{\delta}+1\right) \\
& \quad \times\left(\frac{[r]}{[n+1]}+\frac{1+(q-1)[r]}{[n+1]} t-x\right)\left[\begin{array}{c}
n+p \\
r
\end{array}\right] x^{r} \prod_{s=0}^{n+p-r-1}\left(1-q^{s} x\right) d_{q} t
\end{aligned}
$$

since,

$$
\xi-x \leq \frac{[r]}{[n+1]}+\frac{1+(q-1)[r]}{[n+1]} t-x .
$$

From the Cauchy-Schwarz inequality, for the first term, we get

$$
\begin{aligned}
& \left|K_{n}^{p}(f ; q ; x)-f(x)\right| \\
& \quad \leq M \rho_{n, q}(x) \\
& \quad+\omega\left(f^{\prime}, \delta\right) \sum_{r=0}^{n+p} \int_{0}^{1}\left(\frac{[r]}{[n+1]}+\frac{1+(q-1)[r]}{[n+1]} t-x\right)\left[\begin{array}{c}
n+p \\
r
\end{array}\right] x^{r} \prod_{s=0}^{n+p-r-1}\left(1-q^{s} x\right) d_{q} t
\end{aligned}
$$




$$
\begin{aligned}
+ & \frac{\omega\left(f^{\prime}, \delta\right)}{\delta} \sum_{r=0}^{n+p} \int_{0}^{1}\left(\frac{[r]}{[n+1]}+\frac{1+(q-1)[r]}{[n+1]} t-x\right)^{2}\left[\begin{array}{c}
n+p \\
r
\end{array}\right] x^{r} \prod_{s=0}^{n+p-r-1}\left(1-q^{s} x\right) d_{q} t \\
\leq & M \rho_{n, q}(x) \\
& +\omega\left(f^{\prime}, \delta\right)\left(\sum_{r=0}^{n+p} \int_{0}^{1}\left(\frac{[r]}{[n+1]}+\frac{1+(q-1)[r]}{[n+1]} t-x\right)^{2}\right. \\
& \left.\times\left[\begin{array}{c}
n+p \\
r
\end{array}\right] x^{r} \prod_{s=0}^{n+p-r-1}\left(1-q^{s} x\right) d_{q} t\right)^{1 / 2} \\
& +\frac{\omega\left(f^{\prime}, \delta\right)}{\delta} \sum_{r=0}^{n+p} \int_{0}^{1}\left(\frac{[r]}{[n+1]}+\frac{1+(q-1)[r]}{[n+1]} t-x\right)^{2}\left[\begin{array}{c}
n+p \\
r
\end{array}\right] x^{r} \prod_{s=0}^{n+p-r-1}\left(1-q^{s} x\right) d_{q} t \\
= & M \rho_{n, q}(x)+\omega\left(f^{\prime}, \delta\right) \sqrt{K_{n}^{p}\left((u-x)^{2} ; q ; x\right)}+\frac{\omega\left(f^{\prime}, \delta\right)}{\delta} K_{n}^{p}\left((u-x)^{2} ; q ; x\right) \\
= & M \rho_{n, q}(x)+\omega\left(f^{\prime}, \delta\right)\left(1+\sqrt{\delta_{n, q, p}(x)}\right)
\end{aligned}
$$

where

$$
\begin{aligned}
\delta:= & \delta_{n, q, p}(x)=\left[\left(\frac{4 q^{4}+q^{3}+q^{2}}{[2][3][n+1]^{2}}[n+p-1][n+p]-4 \frac{[n+p]}{[n+1][2]} q+1\right) x^{2}\right. \\
& \left.+\left(\frac{4 q^{3}+5 q^{2}+3 q}{[2][3][n+1]^{2}}[n+p]-\frac{2}{[2][n+1]}\right) x+\frac{1}{[3][n+1]^{2}}\right] .
\end{aligned}
$$

Finally, we have

$$
\left|K_{n}^{p}(f ; q ; x)-f(x)\right| \leq M \rho_{n, q}(x)+\omega\left(f^{\prime}, \delta\right)\left(1+\sqrt{\delta_{n, q, p}(x)}\right) .
$$

This completes the proof.

\section{Concluding remarks}

In this paper, we obtain many results in the pointwise sense. On the other hand, we see that the interval is bounded and closed, and also $f$ is continuous on it, so these results can be given in the uniform sense.

\section{Competing interests}

The authors declare that they have no competing interests.

\section{Authors' contributions}

All authors completed the paper together. All authors read and approved the final manuscript.

Received: 19 November 2012 Accepted: 9 July 2013 Published: 1 October 2013

\section{References}

1. Schurer, F: Linear Positive Operators in Approximation Theory. Math. Inst., Techn. Univ. Delf Report (1962)

2. Barbosu, D: A survey on the approximation properties of Schurer-Stancu operators. Carpath. J. Math. 20, 1-5 (2004)

3. Lupaş, AA: q-Analogue of the Bernstein operators. In: Seminar on Numerical and Statistical Calculus, University of Cluj-Napoca vol. 9, pp. 85-92 (1987)

4. Phillips, GM: On generalized bernstein polynomials. In: Numerical Analysis, vol. 98, pp. 263-269. World Scientific, River Edge (1996)

5. BüyükyazıCı, I, Sharma, H: Approximation properties of two-dimensional q-Bernstein-Chlodowsky-Durrmeyer operators. Numer. Funct. Anal. Optim. 33(2), 1351-1371 (2012)

6. Büyükyazıcı, I, Atakurt, Ç: On Stancu type generalization of q-Baskakov operators. Math. Comput. Model. 52(5-6), $752-759$ (2010) 
7. Gupta, V, Finta, Z: On certain q-Durrmeyer type operators. Appl. Math. Comput. 209(2), 415-420 (2009)

8. Mahmudov, NI, Sabancigil, P: q-Parametric Bleimann Butzer and Hahn operators. J. Inequal. Appl. 2008, Article ID 816367 (2008)

9. Ostrovska, S: q-Bernstein polynomials and their iterates. J. Approx. Theory 123, 232-255 (2003)

10. Wang, H, Wu, XZ: Saturation of convergence for $q$-Bernstein polynomials in the case $q>1$. J. Math. Anal. Appl. 337, 744-750 (2008)

11. Muraru, CV: Note on q-Bernstein-Schurer operators. Stud. Univ. Babeş-Bolyai, Math. 56, 489-495 (2011)

12. Kac, V, Cheung, P: Quantum Calculus. Springer, New York (2002)

13. Vedi, T, Özarslan, MA: Some properties of q-Bernstein-Schurer operators. J. Appl. Funct. Anal. 8(1), 45-53 (2013)

14. Özarslan, MA: q-Szasz Schurer operators. Miskolc Math. Notes 12, 225-235 (2011)

15. Duman, O, Özarslan, MA, Doğru, O: On integral type generalizations of positive linear operators. Stud. Math. 176(1), 1-12 (2006)

16. Duman, O, Özarslan, MA, Vecchia, BD: Modified Szasz-Mirakjan-Kantorovich operators preserving linear functions. Turk. J. Math. 33(2), 151-158 (2009)

17. Özarslan, MA, Duman, O: Global approximation properties of modified SMK operators. Filomat 24(1), 47-61 (2010)

18. Özarslan, MA, Duman, O: Local approximation behavior of modified SMK operators. Miskolc Math. Notes 11(1), 87-99 (2010)

19. Özarslan, MA, Duman, O, Srivastava, HM: Statistical approximation results for Kantorovich-type operators involving some special functions. Math. Comput. Model. 48(3-4), 388-401 (2008)

20. Dalmanoğlu, Ö: Approximation by Kantorovich type q-Bernstein operators. In: MATH'07: Proceedings of the 12 th WSEAS Intenational Conference on Applied Mathematics, Egypt 2007, pp. 29-31 (2007)

21. DeVore, RA, Lorentz, GG: Constructive Approximation. Springer, Berlin (1993)

\section{Submit your manuscript to a SpringerOpen ${ }^{\ominus}$ journal and benefit from:}

- Convenient online submission

- Rigorous peer review

Immediate publication on acceptance

Open access: articles freely available online

- High visibility within the field

- Retaining the copyright to your article 